Check for updates

Cite this: Mater. Chem. Front., 2019, 3, 1661

Received 7th May 2019,

Accepted 11th June 2019

DOI: $10.1039 / c 9 q m 00293 f$

rsc.li/frontiers-materials

\section{Deep insights into polymorphism initiated by exploring multicolor conversion materials $\dagger$}

\author{
Yongtao Wang, (1) *a Wenjing Liu, Litong Ren ${ }^{\mathrm{b}}$ and Guixian Ge*
}

The discovery of mechanochromic materials with multicolor conversion has mainly depended on accidents and good fortune until now, but manipulating molecular aggregation modes to achieve polymorphism is highly efficient. To obtain multicolor conversion materials and explore the intrinsic mechanism of polymorphism, TPEB2F and TPEB3F were designed and synthesized based on molecular isomerism and aggregation-induced emission (AIE) theory. Although they have similar solvatochromism and AIE properties, TPEB2F and TPEB3F show a high-contrast polymorphism and mechanochromism in terms of reversibility, fluorescence emission intensity and wavelength shift. Only crystal $\mathbf{2 R}$ was obtained for TPEB2F, but four different crystals 3Y, 3R, 3N and 3F exist for TPEB3F in the same solvent environment. Emission maxima of $\mathbf{2} \mathbf{R}$ show a $22 \mathrm{~nm}$ red shift, and its quantum yield $\left(\Phi_{\mathrm{F}}\right)$ was significantly increased to 0.37 from 0.09 after hard grinding. 3Y provided a maximum redshift $(40 \mathrm{~nm})$ and slightly reduced $\Phi_{\mathrm{F}}$ due to different intermolecular stacking and arrangement compared to the others, while $3 \mathrm{~N}$ exhibited a remarkable blue shift $(48 \mathrm{~nm})$ and emission enhancement by gently grinding. X-ray single crystal analysis indicated that mechanochromism was not only related to intermolecular stacking and arrangement but also to the crystal system, space group and density. Theoretical calculations revealed that $\mathbf{2 R}, \mathbf{3 Y}$ and $\mathbf{3 R}$ have an intramolecular charge transfer effect, and furthermore, the key to the formation of polymorphism mainly depends on the dipole moment of stacked molecular pairs and culture solvents rather than the minimum energy of stacked molecular pairs and the dipole moment of a single molecule. In addition, the by-product was purified and characterized in detail for the first time.

\section{Introduction}

Mechanochromic materials have attracted increasing attention in recent years owing to their promising potential for applications in mechano-sensors, nondestructive detection, anti-counterfeiting, security papers, memory chips and data storage. ${ }^{1-7}$ Under external stimuli such as ultrasound, grinding, scraping, rubbing or compressing, stretching and shrinking, these mechanochromic materials and their doped polymers exhibit significant changes in terms of emission peak or intensity, and then return to the initial state by solvent fuming and heating. ${ }^{8-11}$ More importantly, the chemical structures of the materials are not destroyed in the above processes. However, our current understanding of the intrinsic mechanism of the materials is mainly derived from

\footnotetext{
${ }^{a}$ Guangxi Key Laboratory of Electrochemical and Magneto-chemical Function Materia, College of Chemistry and Bioengineering, Guilin University of Technology, Guilin 541004, China. E-mail: wyt_shzu@163.com

${ }^{b}$ Shihezi University, Shihezi 832003, P. R. China. E-mail: geguixian@126.com $\dagger$ Electronic supplementary information (ESI) available: ${ }^{1} \mathrm{H}$ NMR, ${ }^{13} \mathrm{C}$ NMR, fluorescence, absorption, transmittance, hydrogen bonding interaction and MALDI-TOF MS spectra [supplemental figures]. CCDC 1912365, 1912366, 1912368, 1912460 and 1912461. For ESI and crystallographic data in CIF or other electronic format see DOI: $10.1039 / \mathrm{c} 9 \mathrm{qm} 00293 \mathrm{f}$
}

analysis and interpretation of their corresponding properties, and it's too soon to give definite design rules. Even so, based on conformation planarization, phase transition of metal-metal interaction, crystal-amorphous, crystal-crystal, amorphouscrystal, crystal-amorphous-crystal and so on, ${ }^{12,13}$ currently, it is no longer difficult to obtain mechanochromic materials with double color conversion, but multicolor switches really depend more on luck and accidental results.

Taking advantage of aggregation induced emission (AIE) theory ${ }^{14}$ and halogen atom effects, ${ }^{15,16}$ hydrophilic and hydrophobic interactions, ${ }^{17}$ intramolecular charge transfer, ${ }^{18-20}$ and so forth, twisted molecular configurations and intermolecular weak bond interactions are constructed. Furthermore, the twisted configurations and weak interactions afforded a relatively loose packing, ${ }^{21}$ which is prone to be compressed and destroyed by exogenous forces and accompanied by fluorescence changes. Subsequently, molecular conformations and intermolecular interactions are reconstructed by solvent fumigation and recrystallization. Then, reversible mechanochromic materials are found. However, few multicolor mechanochromic materials have been reported until now. It is especially noticed that manipulating a crystalline state to realize polymorphism has been an important technique to obtain multicolor mechanochromic materials. Polymorphism maintains the 
same molecular structure, but two or more crystalline phases and shows sharp variations in optical properties. For example, Peng et $a .^{22}$ gained yellow, green and blue luminous aggregates by changing the types and concentrations of surfactants. Wang et $a .^{23}$ cultured four crystals of single luminogens with different colors, while Huang et al. ${ }^{10}$ achieved tricolor crystals by tuning molecular aggregation. Generally, the crystals possess a mechanochromic property due to phase transition of an amorphous crystal. Next, by direct transition of crystal-crystal or indirect transition of crystal-amorphous-crystal, multicolor switches are smoothly realized by taking advantage of polymorphs. Faced with limited quantities, it is difficult to explore the intrinsic mechanism of polymorphs. Based on X-ray crystallographic analysis, an opportunity is provided to correlate mechanochromism with luminescence properties of polymorphs. Not only that, continuous research of polymorphs is also beneficial to understand in-depth metal-free room temperature phosphorescence and enhancement or attenuation plus red-shift or blue-shift of emission wavelengths for mechanochromic materials. More importantly, polymorphs can provide critical examples and information, deep analysis and thinking for definite structure-packing-performance. ${ }^{24}$

To obtain polymorphs and explore their corresponding internal mechanisms, in this paper, two organic boron complexes, named as TPEB2F and TPEB3F, were designed and synthesized. Both luminogens were furnished with a propeller-like tetraphenylethylene (TPE) unit to maintain a twisted molecular conformation, while isomerism effects were investigated by changing the substitution position of furan, which has been rarely reported in polymorphs. Based on the slight structural differences and resulting high-contrast photophysical properties, it is conducive to further discussion and understanding of the inherent mechanisms of polymorphs and mechanochromism. As expected, TPEB3F has four different crystal forms named as $\mathbf{3 Y}, \mathbf{3 R}, \mathbf{3 N}$ and $\mathbf{3 F}$. More interestingly, $\mathbf{3 R}(48 \mathrm{~nm})$ and $\mathbf{3 N}(26 \mathrm{~nm})$ show remarkable blueshifted and enhanced emission after slight grinding, but the emission maxima of $\mathbf{2 R}$ exhibit a faint red shift. After hard grinding, 3Y shows the biggest red shift among the crystals, followed by $\mathbf{2} \mathbf{R}$ and $\mathbf{3 F}$, while $\mathbf{3 R}$ and $\mathbf{3 N}$ still maintain a blue shift. Combining crystal analysis, photophysical properties and theoretical calculations, the effects of molecular isomerism on polymorphism and mechanochromism were analyzed and discussed in depth. In addition, a scarce by-product was isolated and purified in the preparation process of the two complexes, and the molecular structure of the by-product was characterized by NMR, HRMS and single crystal diffraction.

\section{Results and discussion}

\subsection{Synthesis and characterization}

The two $\beta$-diketonates were prepared by a condensation reaction between $\mathbf{C H}_{3}$ COTPE and $2 \mathbf{F}$ or $3 \mathbf{F}$ at $60{ }^{\circ} \mathrm{C}$, then cyclized with $-\mathrm{BF}_{2}$ to yield TPEB2F and TPEB3F. The different ratios of raw materials were investigated in the condensation reaction (Scheme 1). When the ratio of $\mathbf{C H}_{3} \mathbf{C O T P E}: \mathbf{N a H}: 3 \mathbf{F}$ is $1: 2: 1.5$, then thin layer chromatography (TLC) indicated the reaction solution contained two products emitting orange (main product with an AIE effect) and blue fluorescence (by-product with an aggregation caused quenching effect) respectively, under an ultraviolet lamp. Adjusting the reaction ratio to $\mathbf{C H}_{3} \mathbf{C O T P E}: \mathbf{N a H}: 3 \mathbf{F}=1: 1.2: 3$, the by-product decreased significantly with an increase of main product. Furthermore, the by-product was characterized by NMR and $\mathrm{X}$-ray single crystal analysis (Fig. 1 and Fig. S9, S10, ESI $\dagger$ ), indicating generation of the by-products is related to the reduction of $\mathrm{NaH}$, which leads to hydrogenation of unsaturated double bonds. Single crystal analysis shows that the by-product (CCDC 1912231†) adopts approximate planar molecular configurations and anti-parallel head-to-head stacking, forming $3.62^{\circ}$ and $4.96^{\circ}$ dihedral angles between planes $A, C$ and $B$ respectively, with $3.591 \AA$ centroid-centroid $\left(\mathrm{C}_{\mathrm{g}}-\mathrm{C}_{\mathrm{g}}\right)$ distances between furan and boron heterocycles, as well as $3.870 \AA \mathrm{C}_{\mathrm{g}}-\mathrm{C}_{\mathrm{g}}$. distances between boron heterocycles (Fig. 1). Obviously, the stacking modes and planar molecular configurations should be responsible for its fluorescence quenching in the aggregation state.

\subsection{Photo-physical properties in solution}

Photo-physical properties of the two complexes in various organic solvents were investigated and the results and data are shown in Fig. 2 and Table S1 (ESI $\dagger$ ). When the polarity was increased from hexane to dimethyl sulfoxide, the absorption maxima of TPEB2F and TPEB3F were red-shifted by $16 \mathrm{~nm}$ and $11 \mathrm{~nm}$, respectively. Compared with absorption spectra, the emission maxima exhibit remarkable bathochromic shifts and obvious solvatochromic behavior, hinting of an easily polarized excitation state and intramolecular charge transfer (ICT) effect.

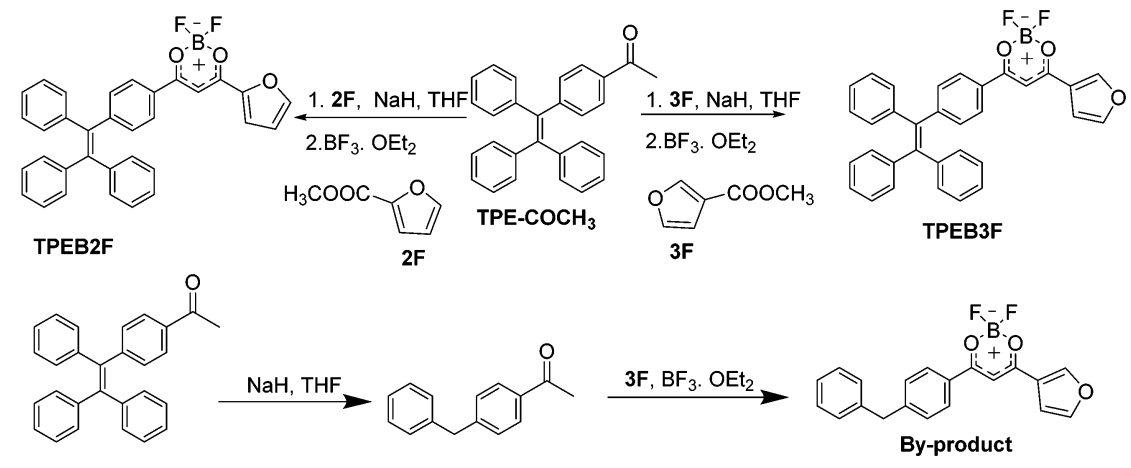

Scheme 1 Synthetic routes of TPEB2F, TPEB3F and by-product. 


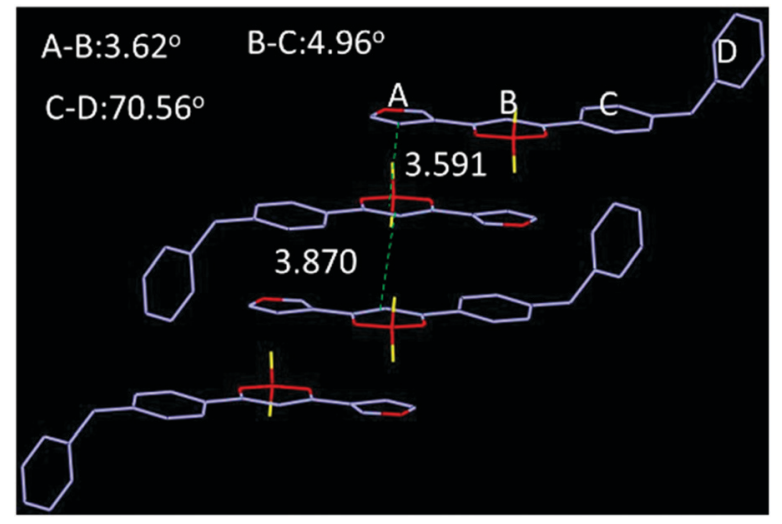

Fig. 1 Molecular configurations and intermolecular stacking modes of by-product without hydrogen.

For TPEB2F, not only absorption but also emission maxima appeared shifted towards the longer wavelength side compared to that of TPEB3F in solution, indicating an isomerism effect on molecular conjugation, which is likely due to better conjugation or stronger electron-donor effects of the $\alpha$-position than $\beta$-position for furan.

\subsection{Aggregation induced emission}

Pristine power of the two luminogens have strong emissions under excitation of $356 \mathrm{~nm}$, but their fluorescence's are faint in dilute solution to the naked eye, which present a high contrast to the AIE scene. Further, their fluorescence emission spectra were investigated in THF/water solutions with different water fractions (Fig. 3). As the volume fraction of water $\left(f_{\mathrm{w}}\right)$ in the mixed solvent continued to increase to $70 \%$, the weak fluorescence emission did not have significant change due to unimolecular dispersion in the dissolved state. Significant fluorescence enhancement induced by aggregation occurs at $f_{\mathrm{w}}=80 \%$, reaching a maximum at $f_{\mathrm{w}}=90 \%$, thereby the intramolecular rotation and vibration are suppressed. These results again verify propeller-like TPE unit can effectively improve luminescence of aggregated states. More interestingly, there is almost no obvious difference for the two dyes in AIE properties and emission maxima.

\subsection{Mechanofluorochromic properties}

According to literature reports, the twisted molecular configuration and crystalline state support loose molecular stacking and ordered molecular arrangement respectively, while phase transition from crystalline to amorphous leads to disorder and collapse of the molecular arrangement and stacking under external force, which thereby triggers mechanofluorochromism. ${ }^{25}$ To investigate mechanochromic properties of TPEB2F and TPEB3F in depth, we successfully cultured and obtained five different crystals by slow evaporation of a DCM/hexane mixed solvent. The wonder is that only one orange crystal (2R) was obtained for TPE2F, but TPEB3F provided four crystals $(\mathbf{3 Y}, \mathbf{3 R}, \mathbf{3 N}$ and $\mathbf{3 F}$ ) despite
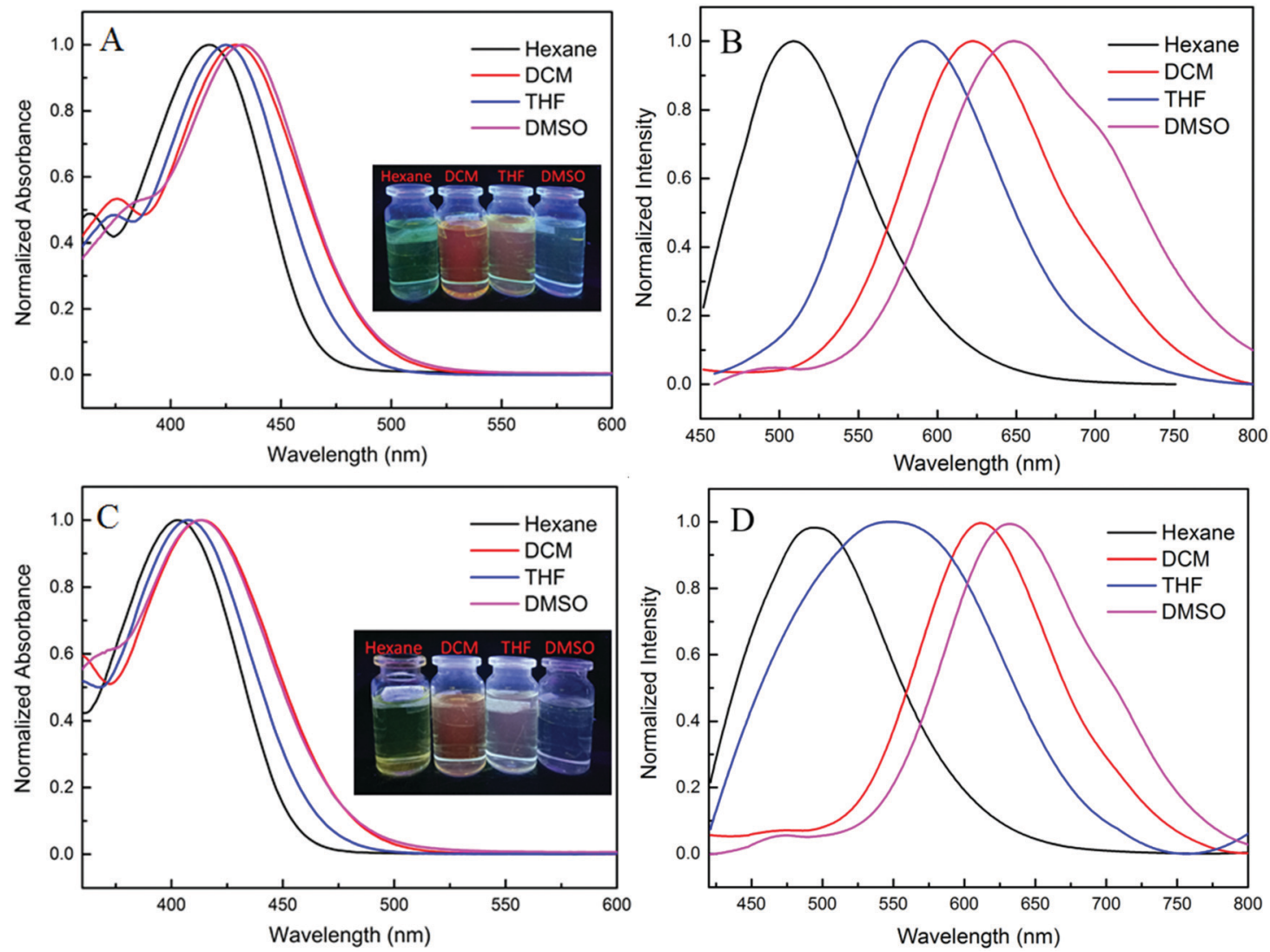

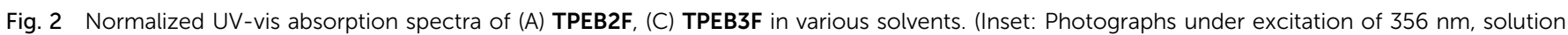
concentration: $10 \mu \mathrm{M}$.) Normalized fluorescence spectra of (B) TPEB2F, (D) TPEB3F in various solvents (solution concentration: $10 \mu \mathrm{M}$ ). 

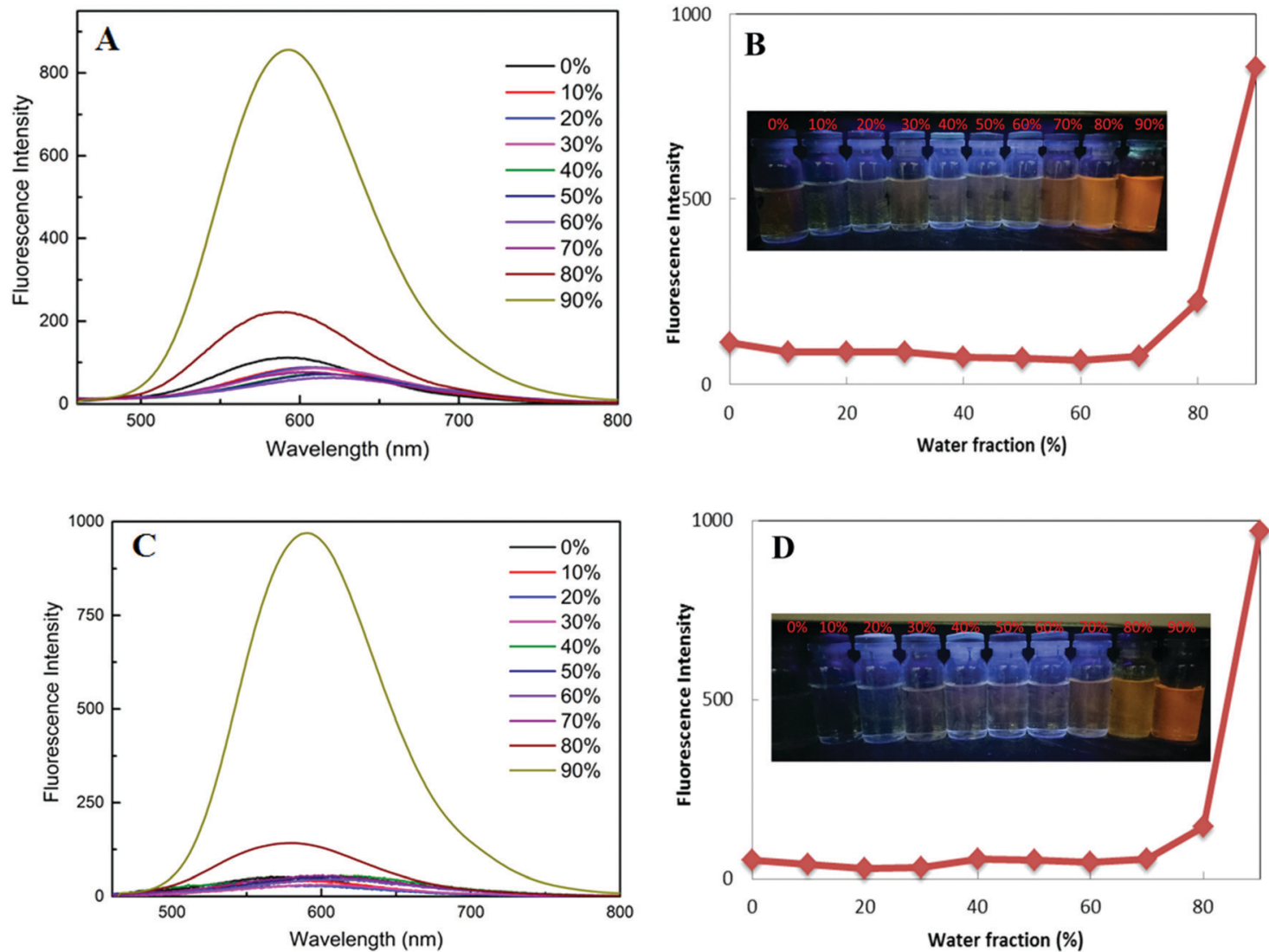

Fig. 3 Fluorescence emission spectra of (A) TPEB2F, (C) TPEB3F in the THF/water mixtures with different fractions of water. Solution concentration: $10 \mu \mathrm{M}$. Chart of relationship between FL peak intensity and water fraction for (B) TPEB2F, (D) TPEB3F. Solution concentration: $10 \mu \mathrm{M}$. Inset: Photographs in THF/water mixtures with different fractions of water under $365 \mathrm{~nm}$ UV illumination.

similar chemical structures and the same culture condition. The fluorescence emission peaks of $2 \mathbf{R}, 3 \mathbf{Y}, 3 \mathbf{R}, 3 \mathbf{N}$ and $3 \mathbf{F}$ are located at 586, 547, 580, 617 and $602 \mathrm{~nm}$, respectively (Table S2, ESI $\dagger$ ). By naked eye observation, the red crystals $\mathbf{3 R}, \mathbf{3 N}$ and $\mathbf{3 F}$ display particle, needle-like and block shapes in sequence; further, the different morphologies are distinguished by fluorescence microscopy, indicating cluster $\mathbf{3 R}$, cubic $\mathbf{3 N}$ and layered structure $\mathbf{3 F}$ (Fig. 4). Obviously, TPEB3F crystals compared with TPEB2F have more chance to exhibit mechanochromic luminescence due to four aggregation states. More significantly, only a few emitters show both mechanochromism and polymorphism fluorescence until now. ${ }^{26,27}$ To demonstrate mechanochromic luminescence and gain a multicolor switch, fluorescence emission spectra of the crystals were studied by slight and hard grinding, heating and solvent fuming. As shown in Fig. 5, 6 and Table S2 (ESI $\dagger$ ), 2R underwent an obvious red shift from $586 \mathrm{~nm}$ to $608 \mathrm{~nm}$ and enhanced fluorescence emission with fluorescence quantum efficiency from 9.27 to 36.67 after hard grinding, and returned to the emission band of the crystal

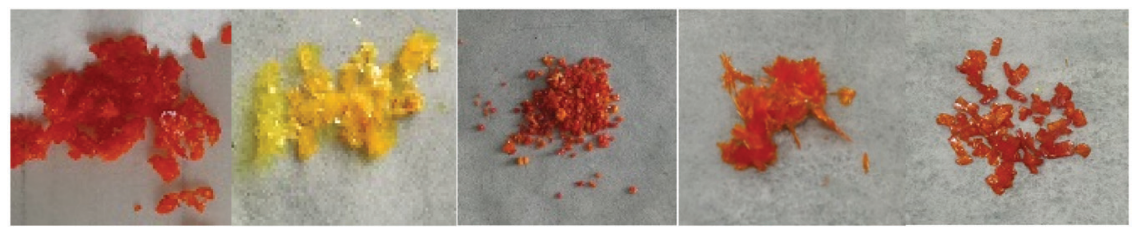

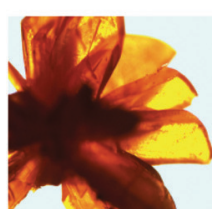

2R

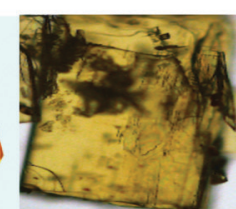

$3 Y$

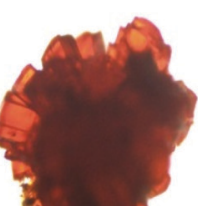

3R

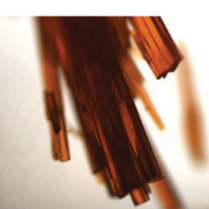

3N

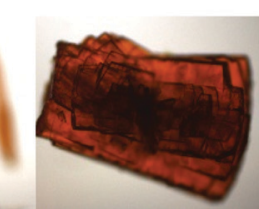

$3 \mathbf{F}$

Fig. 4 Photographs of the five crystals of TPEB2F and TPEB3F under natural light (first row), photographs of the five crystals under a fluorescence microscope (second row); from left to right, are $\mathbf{2 R}, \mathbf{3 Y}, \mathbf{3 R}, \mathbf{3 N}, \mathbf{3 F}$, respectively. 


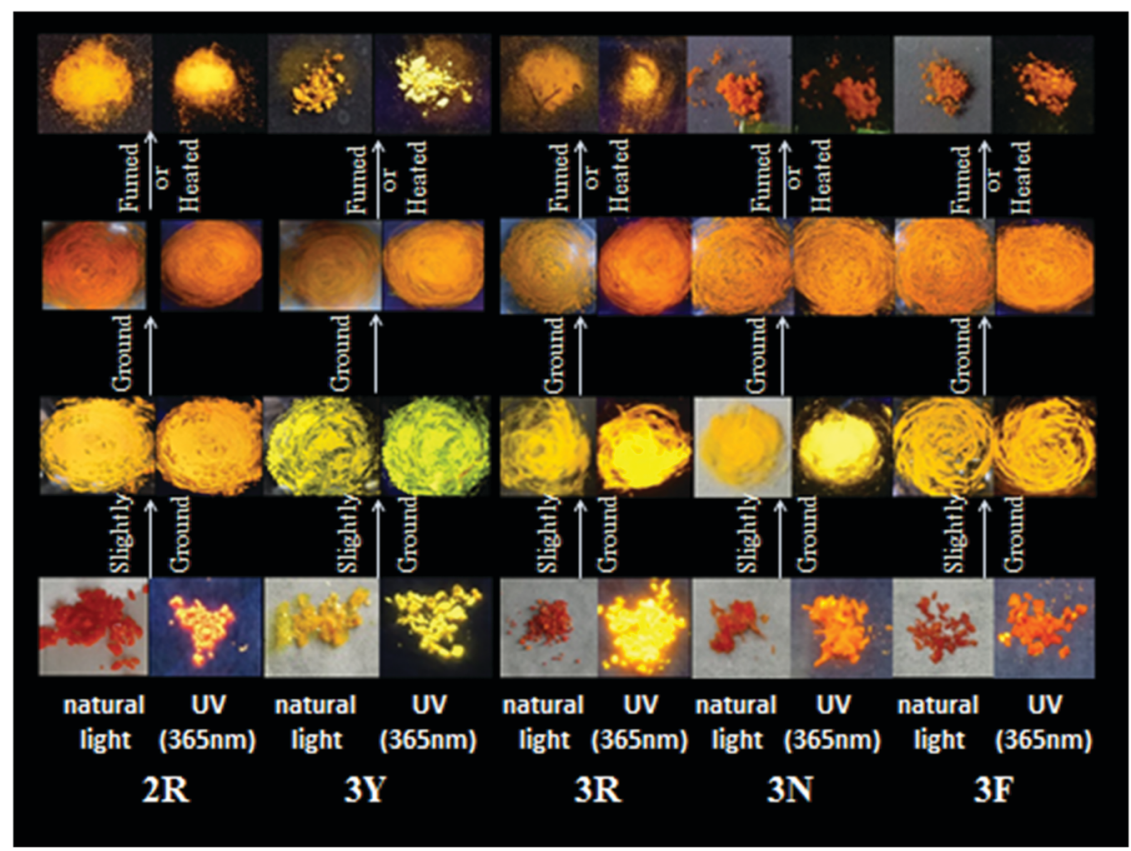

Fig. 5 Photographic images of TPEB2F and TPEB3F in different solid states under natural light and UV light (365 nm).

after fuming or heating the ground samples at $150{ }^{\circ} \mathrm{C}$ for 10 minutes; however, it was insensitive to weak mechanical forces and showed $3 \mathrm{~nm}$ red-shift after slight grinding. The reversible mechanochromism shows solvent molecules and heating can promote molecules rearranging and returning to their original phase. The red shifted fluorescence emission may be due to conformational planarization and closer molecular packing caused by external force, which inhibit vibration and rotation of bonds and induce enhanced emission. More importantly, crystal $3 Y$ relative to the others exhibited higher contrast after hard grinding, and $40 \mathrm{~nm}$ redshift was observed after slight grinding. Different from 2R, the mechanochromism of 3Y, 3R, 3N and 3F is irreversible whether it is heated or fumigated, showing an obvious isomerization effect. More interesting, 3Y, 3R, 3N and 3F exhibited significant color changes after slightly grinding, and the emission color changed from red to yellow and the brightness was obviously enhanced under ultraviolet light, which conforms to the energy gap rule. Especially for $\mathbf{3 N}$, such large blue-shifts (48 $\mathrm{nm}$ ) and enhanced emissions with quantum efficiencies from 1.63 to 9.26 are rare in previously reported mechanochromic materials. According to literature reports, a blue shift is usually attributed to the following three aspects: the first is the destruction of intermolecular weak forces maintaining planar conformation by mechanical forces, producing more twisted molecular conformations. ${ }^{28}$ The second is the opposite process of aggregation named as "disaggregation", leading to blue-shift of emission wavelength and enhancement of emission intensity. ${ }^{29}$ The third is grinding altered "electron delocalization" and band gap due to reduced particle diameters, which causes short emission wavelength. ${ }^{30}$ We think the blue-shift is related to the collapse of J-type stacking. But the question is, how to enlarge contrast of fluorescence emission before/after slight grinding? These explanations are still powerless. Most crystals are insensitive to weak mechanical forces and affect detection limits of piezo-luminescence sensors; thus, $\mathbf{3 N}$ is very precious and expected to make up for the shortage of weak mechanical forces sensors. Moreover, the emission wavelength of $\mathbf{3 N}$ presents significantly decreasing shifts after hard grinding, which further confirmed the unique sensor performance of weak mechanical forces for $\mathbf{3 N}$. Meanwhile, 3Y, 3R, 3N and 3F show obvious tricolor emissions from yellow to red by different grinding degrees, which are clearly visible to the naked eyes due to different emission intensities and shifts. In principle, hard grinding powders from 3Y, 3R, 3N and $\mathbf{3 F}$ should have the same color and emission color due to the same molecular structures, however, fluorescence emission spectra of the ground samples are distinctly different, especially, emission maxima of $\mathbf{3 F}$ relative to $\mathbf{3 R}$ which shows a bathochromic-shift of up to $44 \mathrm{~nm}$. First, grinding is an anisotropic force and difficult to quantify. Secondly, the crystals have different intermolecular stacking, arrangement, density and melting points (Fig. S1, ESI $\dagger$ ). The samples show different cold crystallization temperatures or glass transition temperatures after grinding, which indicates that the ground samples are really different. As a speculation, the unquantifiable mechanical force, different intermolecular packing, interactions and density easily lead to local distinctions in intermolecular interactions and packing, thereby producing different fluorescence. Furthermore, the ground samples cannot recover their origin color after fuming or heating (Table S2, ESI $\dagger$ ). To further verify the irreversible mechanochromism, rewritable papers were prepared by suspending the powder of TPEB3F in $n$-hexane and spreading it evenly on filter paper (Fig. 7). After the solvent was evaporated, a Chinese knot was drawn on the filter paper, which clearly represented the mechanochromic behaviors. The Chinese knot could not be restored to the original color of the powder by solvent fumigation and heating, which indicates that TPEB3F is suitable for permanent preservation materials. 

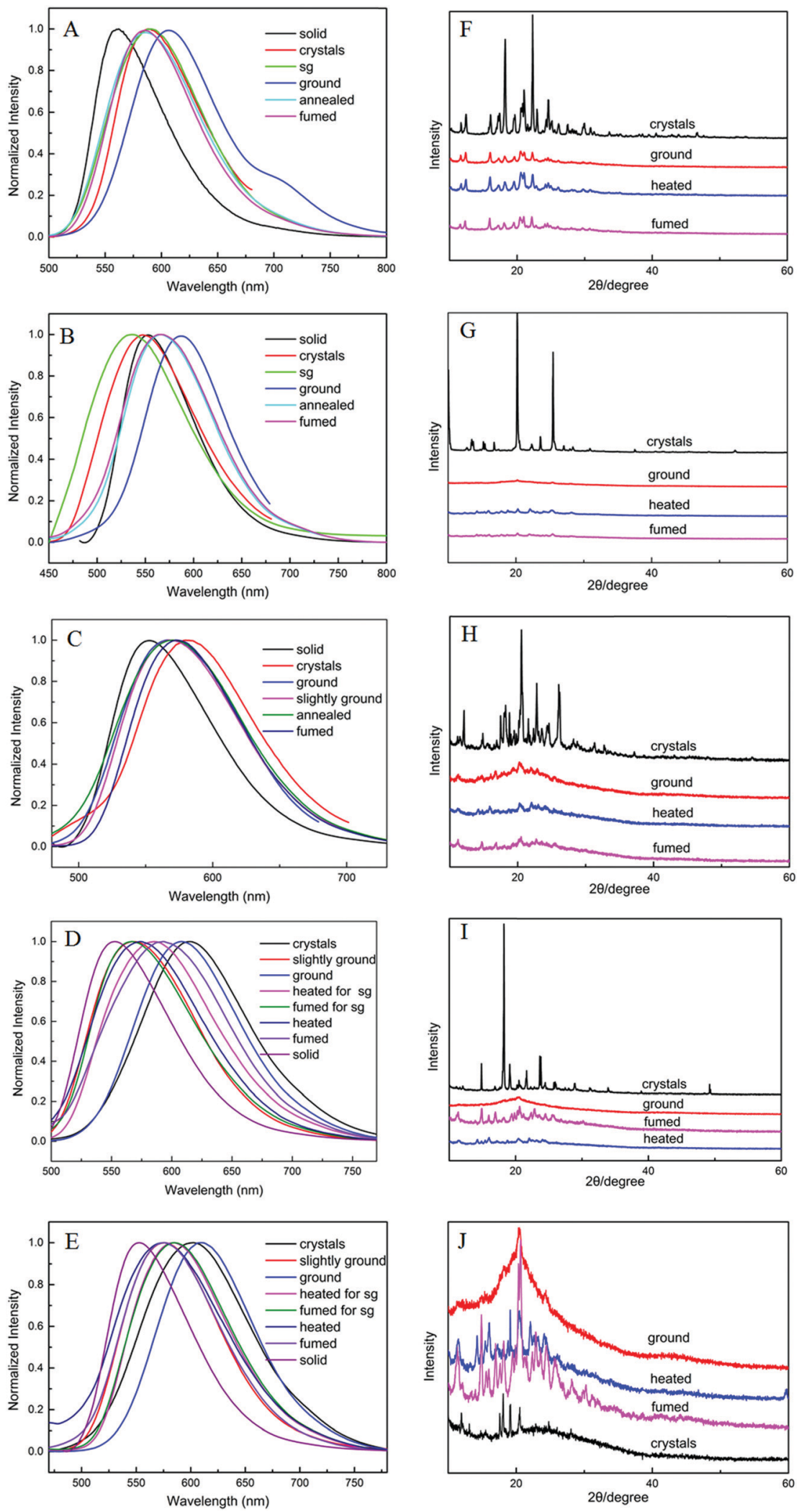

Fig. 6 FL spectra of complexes (A) 2R, (B) 3Y, (C) 3R, (D) 3N, (E) 3F in different solid states, and XRD patterns of (F) 2R, (G) 3Y, (H) 3R, (I) 3N, (J) 3F in various solid states (solid: original state of compounds, sg: slightly ground).

To investigate the internal mechanism of mechanochromism, X-ray diffraction (XRD) (Fig. 6) and differential scanning calorimetry
(DSC) (Fig. S1, ESI $\dagger$ ) tests were conducted. XRD patterns of crystal 2R show sharp and intense reflections, indicating a well-ordered 


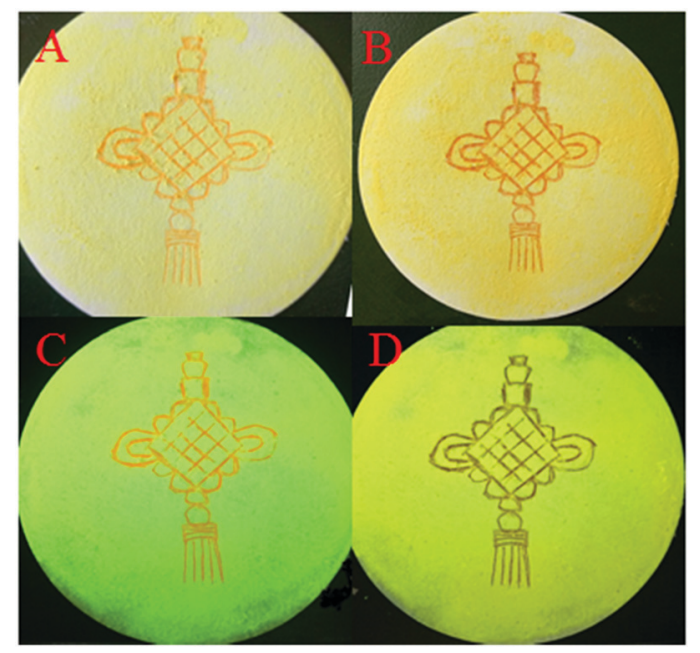

Fig. 7 Photographed with Chinese knot on filter paper with TPEB3F powder dispersion (A) in daylight and (C) in UV light. (B) and (D) are photos from fuming with anhydrous ethanol treatments in daylight and UV light, respectively.

microcrystalline structure. Compared to crystals, diffraction peaks of ground $\mathbf{2} \mathbf{R}$ attenuate or even disappear. In addition, some inconspicuous reflections can partial reappear by fumigation or heating. The XRD patterns indicate TPEB2F has reversible mechanochromism. By contrast, crystals 3Y, 3R, 3N and $\mathbf{3 F}$ show different diffraction peaks indicating different molecular packing. After grinding, diffraction peaks of $\mathbf{3 Y}, \mathbf{3 R}$ and $\mathbf{3 N}$ almost completely disappeared, while $\mathbf{3 F}$ exhibited a wide and large diffraction peak. With further heating and grinding, only 3F displayed strong diffraction peaks, but they were sharply different with primitive crystal diffraction peaks. Therefore, TPEB3F has irreversible mechanochromism probably due to weak crystallization ability. Then, DSC curves further confirmed the different molecular arrangements in different aggregation states. The crystalline samples of $\mathbf{2 R}, \mathbf{3} \mathbf{Y}, \mathbf{3 R}$ and $\mathbf{3 N}$ showed sharp melting peaks, while cold-crystallization transition peaks $\left(132{ }^{\circ} \mathrm{C}, 119{ }^{\circ} \mathrm{C}\right.$ and $\left.126{ }^{\circ} \mathrm{C}\right)$ were present before melting in ground samples, which is attributed to a meta-stable amorphous phase converting into a more stable crystalline phase in the exothermal recrystallization process. More interestingly, crystals 3R and 3N have two different melting peaks at about $240{ }^{\circ} \mathrm{C}$ and $250{ }^{\circ} \mathrm{C}$, which may be attributed to polymorphism of a single crystal or two crystals containing different particle sizes; however, crystal analysis revealed only one pattern of molecular stacking and arrangement exists, thus we hypothesized it should be the latter. In addition, there is an endothermic peak assigned as glass transition temperature at $185{ }^{\circ} \mathrm{C}$ for ground $3 \mathrm{R}$. In general, XRD and DSC investigations can give some reasonable explanations to mechanochromism, but deep understanding such as mechanochromism and polymorphism need to be further explored by crystal analysis and theoretical calculations.

\subsection{Crystal structures}

As shown in Fig. 8, 2R (CCDC 1912365 †), 3Y (CCDC 1912366†) and 3R (CCDC 1912368†) exhibit twisted molecular configurations by

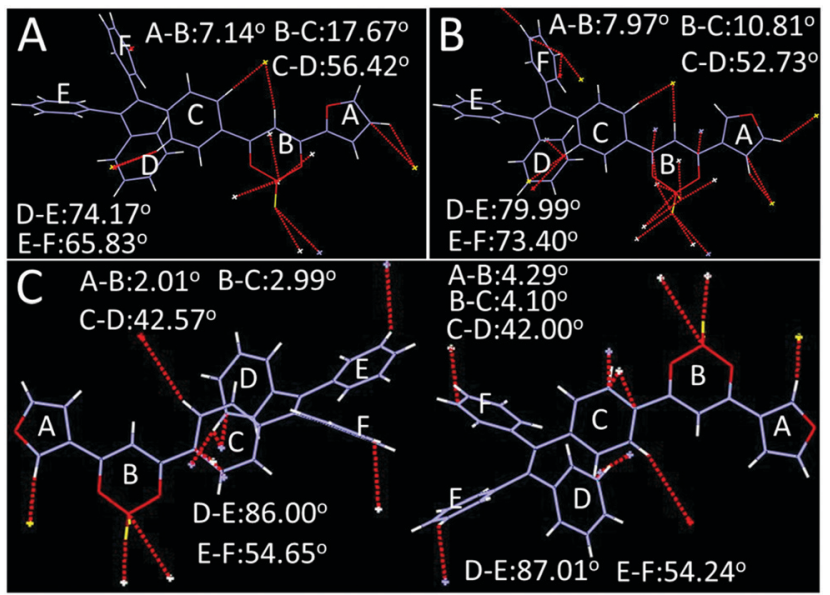

Fig. 8 Single crystal structure and conformation with hydrogen bond and weak interaction sites (A) 2R, (B) 3R, (C) 3 Y.

furnishing propeller-like TPE units and forming various intermolecular hydrogen bonds and weak interactions such as $\mathrm{C}-\mathrm{H} \cdots \mathrm{F}, \mathrm{C}-\mathrm{H} \cdots \mathrm{B}, \mathrm{C}-\mathrm{H} \cdots \mathrm{C}, \mathrm{C} \cdots \mathrm{C}$ and $\mathrm{C} \cdots \mathrm{F}$ (Fig. S2, ESI $\dagger$ ). Similar to previous studies, boron heterocycle (B ring) and 1,3-disubstituted benzene ( $\mathrm{C}$ ring) and furan (A ring) display a V-shape and near-planar configuration, thereby resulting in small steric hindrance and facilitating the molecules adopting a head-to-head stacking mode. Interestingly, the oxygen atom of furan is located on the same side of the boron atom for $\mathbf{3 Y}$, but on two sides for $\mathbf{2 R}$ and $\mathbf{3 R}$. Further, the different locations may affect intermolecular stacking and dipole moments. As expected, crystal analysis revealed the adjacent molecules of $2 \mathbf{R}$ and $3 \mathbf{R}$ form the intermolecular partial overlap between boron heterocycles, while the overlap of $3 \mathbf{Y}$ occurs between the furan ring and boron heterocycle. Meanwhile, 2R and 3R compared with $\mathbf{3 Y}$ possess a larger dihedral angle between the $\mathrm{B}$ ring and $\mathrm{C}$ ring. The twisted structure facilitates the separation of HOMO and LUMO and increases the intramolecular charge transfer effect, thereby producing a red shifted fluorescence emission compared with that of 3Y (Fig. 9). In addition, the two stacking molecular pairs show different molecular conformations in 3Y, which emerges as two $\mathrm{C}_{\mathrm{g}}-\mathrm{C}_{\mathrm{g}}$ distances between planes $A$ and $B(3.729 \AA$ and $3.563 \AA$ ) (Fig. S3, ESI $\dagger$ ) and triggers bigger widths of the half peak in a crystalline state compared with original solid (Fig. 6B). Generally, mechanochromism depends on the intermolecular

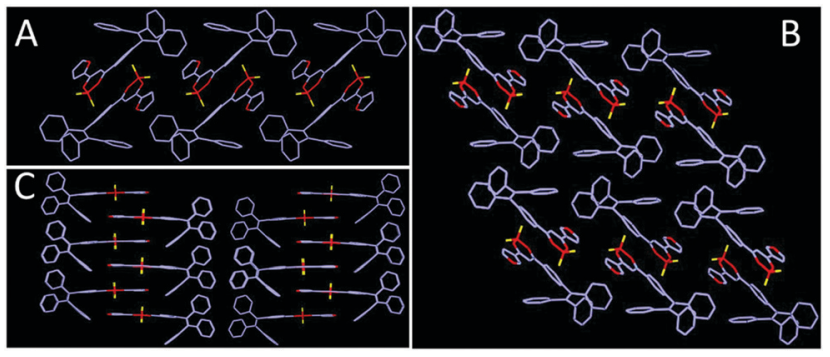

Fig. 9 Intermolecular stacking modes ignoring hydrogen atoms (A) $\mathbf{2}$, (B) $3 R,(C) 3 Y$ 


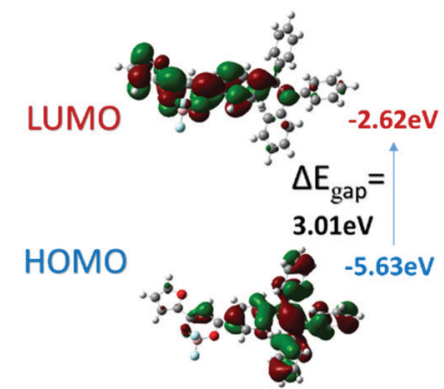

$2 \mathrm{R}$

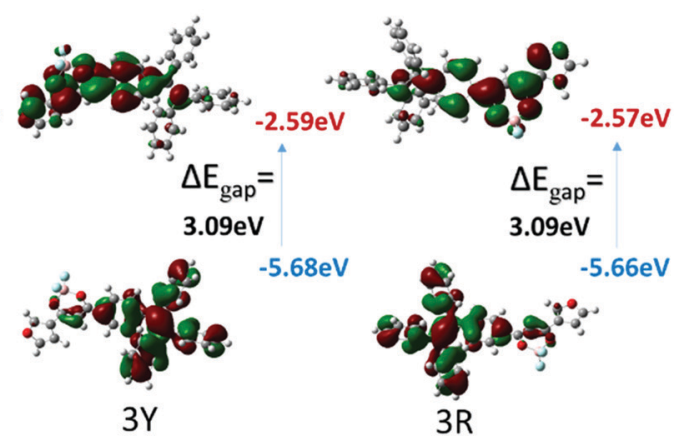

Fig. 10 B3LYP/6-31G(d,p) calculated molecular orbital amplitude plots of HOMO and LUMO levels for 2R, 3Y and 3R.

arrangement and stacking. However, what is puzzling is that $\mathbf{2 R}$ and $\mathbf{3 R}$ with a similar arrangement and stacking give red shifted and blue shifted fluorescence emission respectively, after grinding. Crystal data indicate $2 \mathbf{R}$ is a triclinic system with the space group $P \overline{1}[a=8.788(4) \AA, b=10.446(4) \AA$, and $c=15.407(6) \AA ; \alpha=$ $70.818(10)^{\circ}, \beta=88.67(1)^{\circ}$, and $\left.\gamma=79.808(10)^{\circ}\right]$, while 3Y, 3R, 3N (CCDC 1912460†) and 3F (CCDC 1912461†) with monoclinic systems show hypochromic fluorescence emission after slight grinding. Crystals 3R, $\mathbf{3 N}$ and $\mathbf{3 F}$ are almost identical molecular arrangements and conformations and show similar optical properties. But why does $\mathbf{3 N}$ exhibit the biggest blue shift? In previous studies, we find luminogens with large pore structures exhibit sharp blue-shifts after crushing crystals. Crystal densities of $3 \mathbf{R}, 3 \mathbf{N}$ and $3 \mathbf{F}$ are $1.314,1.307$ and 1.310 in turn, while the degree of blue shift is $\mathbf{3 N}>\mathbf{3} \mathbf{F}>\mathbf{3 R}$, meaning luminogens with smaller density show a bigger shift, obviously, which is consistent with the previous results. However, the wavelength shifts are still irregular after hard grinding. Crystal analyses further indicate $\mathbf{2 R}$ and $\mathbf{3 Y}$ compared with $\mathbf{3 R}, \mathbf{3 N}$ and $\mathbf{3 F}$ have less overlap and weak interactions and larger distances between adjacent $B$ planes, leading to a less repulsive force between $B$ planes (Fig. S2, ESI $\dagger$ ). Hard grinding gives access to approach and overlap of $B$ planes, and intramolecular charge transfers are enhanced. On the contrary, for $\mathbf{3 R}, \mathbf{3 N}$ and $\mathbf{3 F}$, intermolecular distances of $B$ planes are prone to offset rather than compression due to strong interaction, then decreasing $\pi-\pi$ interactions cause a blue shift of emission wavelength. However, the bathochromic-shift of $3 \mathbf{F}$ was an exception, which illustrates grinding as an anisotropic force is complicated for mechanochromism.

\subsection{Theoretical calculations}

To confirm intramolecular charge transfer effects of the two complexes, density functional theory (DFT) calculations on a single molecule were conducted employing the Gaussian 09W program package and DFT/B3LYP/6-31G basis set by extracting data from crystals 2R, 3Y and 3R. Fig. 10 shows HOMO is mainly located on the TPE and $\beta$-diketone boron moiety and the LUMO is mainly distributed on furan and $\beta$-diketone boron units, outlining the obvious shift of electron cloud density.

Theoretically, the existence forms of crystals were related to minimum energy of stacking molecular pairs, while polymorphism was usually obtained by adjusting the polarity of solvents. To further explore the intrinsic mechanism of polymorphism, the minimum energy and dipole moment of stacking molecular pairs extracted from single crystals were completed with the B3LYP/6-31G* basis set using Gaussian 09. Meanwhile, hypothetical crystals 2Ya-d (Table S3 and Fig. S4, ESI $\dagger$ ) were constructed by referring to 3Y and considering isomerism between the boron atom and oxygen atom in a furan ring. As a result, not only TPEB2F but also TPEB3F have almost the same the minimum energy in their respective crystals; obviously, stability is not the main reason of the absence of 2Ya-d. But even more amazing is all the crystals obtained from this experiment possess smaller dipole moments compared with 2 Ya-d, speculating closely related dipole moments exist between stacking molecular pairs and culture solvents. As far as we know, the dipole moments of mixed dichloromethane and $n$-hexane solvents is less than $1.60 \mathrm{D}$. However, the dipole moments of the hypotheticals, except 2Ya, are far beyond $1.6 \mathrm{D}$; interestingly, the maxima of dipole moments of $2 \mathbf{R}, 3 \mathbf{R}, 3 \mathbf{N}, 3 \mathbf{F}$ and $3 Y$ are 0.227 D, far below 1.60 D. Probably, obtaining the hypotheticals with large dipole moments need to increase dipole moments of culture solvents. To further validate the speculation, dipole moments of four single molecules were also calculated. The results show that $\mathbf{2 R}, \mathbf{3 R}$ and $\mathbf{3 Y}$ have similar dipole moments with hypothetical 2Ya, moreover, these similar data are much larger than the dipole moments of culture solvents.

\section{Conclusions}

In summary, two organic boron complexes were designed and synthesized based on AIE effects of the TPE unit and isomerization effects of furan. The reaction process was optimized by changing the mole ratios of the starting materials; moreover, a by-product was characterized by NMR, HR-MS, and single crystal X-ray diffraction, which is attributed to reduction of the ethylene bond induced by $\mathrm{NaH}$. The two luminogens exhibit similar solvatochromism and AIE properties but different mechanochromism and polymorphism. Crystal 2R shows a $22 \mathrm{~nm}$ red shift and elevated $\Phi_{\mathrm{F}}$ from $9.27 \%$ to $36.67 \%$ after hard grinding and comes back to the initial crystalline state with heating and solvent fumigation, but it is insensitive to weak forces. In the same culture medium, 3Y, 3R, 3N and 3F are obtained for TPEB3F and exhibit irreversible mechanochromism. More importantly, the fluorescence emission peak of ground $\mathbf{3 N}$ exhibits a remarkable $48 \mathrm{~nm}$ blue shift after 
slight grinding, while 3Y shows a maximum redshift (40 nm) among the crystals due to different intermolecular stacking and arrangement. The results of XRD and DSC reveal that the intrinsic mechanism of mechanochromism is due to the phase transition from crystalline to amorphous transitions. X-ray single crystal analysis indicated that mechanochromism is not only related to intermolecular stacking and arrangement, but also to the crystal system, space group and crystal density. Theoretical calculations confirmed that polymorphism depends on the dipole moment of stacked molecular pairs and culture solvents rather than the minimum energy of stacked molecular pairs and dipole moments of single molecules.

\section{Conflicts of interest}

There are no conflicts to declare.

\section{Acknowledgements}

This work was supported by the National Natural Science Foundation of China (Grant No. 21766030, 21566034, 21403144 and 11464038).

\section{References}

1 P. Shi, Y. Duan, W. Wei, Z. Xu, Z. Li and T. Han, A turn-on type mechanochromic fluorescent material based on defectinduced emission: implication for pressure sensing and mechanical printing, J. Mater. Chem. C, 2018, 6, 2476-2482.

2 Z. Yang, Z. Chi, Z. Mao, Y. Zhang, S. Liu, J. Zhao, M. P. Aldred and Z. Chi, Recent advances in mechano-responsive luminescence of tetraphenylethylene derivatives with aggregationinduced emission properties, Mater. Chem. Front., 2018, 2, 861-890.

3 Y. Hou, J. Du, J. Hou, P. Shi, K. Wang, S. Zhang, T. Han and Z. Li, Rewritable optical data storage based on mechanochromic fluorescence materials with aggregation-induced emission, Dyes Pigm., 2019, 160, 830-838.

4 H. Sun, S. Liu, W. Lin, K. Y. Zhang, W. Lv, X. Huang, F. Huo, H. Yang, G. Jenkins, Q. Zhao and W. Huang, Smart responsive phosphorescent materials for data recording and security protection, Nat. Commun., 2014, 5, 3601.

5 H. Zhao, Y. Wang, Y. Wang, G. He, M. Xue, P. Guo, B. Dai, Z. Liu and Y. Qi, Correction: AIE-active mechanochromic materials based $\mathrm{N}$-phenylcarbazol-substituted tetraarylethene for OLED applications, RSC Adv., 2015, 5, 28190.

6 C. Ge, Y. Liu, X. Ye, X. Zheng, Q. Han, J. Liu and X. Tao, Dicyanopyrazine capped with tetraphenylethylene: polymorphs with high contrast luminescence as organic volatile sensors, Mater. Chem. Front., 2017, 1, 530-537.

7 H. Zhao, Y. Wang, Y. Wang, G. He, M. Xue, P. Guo, B. Dai, Z. Liu and Y. Qi, AIE-active mechanochromic materials based N-phenylcarbazol-substituted tetraarylethene for OLED applications, RSC Adv., 2015, 5, 19176-19181.
8 Z. Ma, Z. Wang, M. Teng, Z. Xu and X. Jia, Mechanically induced multicolor change of luminescent materials, ChemPhysChem, 2015, 16, 1811-1828.

9 X. Zhang, Z. Chi, Y. Zhang, S. Liu and J. Xu, Recent advances in mechanochromic luminescent metal complexes, J. Mater. Chem. C, 2013, 1, 3376.

10 B. Huang, W. C. Chen, Z. Li, J. Zhang, W. Zhao, Y. Feng, B. Z. Tang and C. S. Lee, Manipulation of molecular aggregation states to realize polymorphism, AIE, MCL, and TADF in a single molecule, Angew. Chem., Int. Ed., 2018, 57, 12473-12477.

11 Y. Qi, W. Liu, Y. Wang, L. Ma, Y. Yu, Y. Zhang and L. Ren, The inherent mechanism of mechanochromism under different stress: electron cloud density distribution, J-type stacking, pore structure and collapse of J-type stacking, New J. Chem., 2018, 42, 11373-11380.

12 M. Tanioka, S. Kamino, A. Muranaka, Y. Ooyama, H. Ota, Y. Shirasaki, J. Horigome, M. Ueda, M. Uchiyama, D. Sawada and S. Enomoto, Reversible near-infrared/blue mechanofluorochromism of aminobenzopyranoxanthene, J. Am. Chem. Soc., 2015, 137, 6436-6439.

13 P.-Z. Chen, H. Zhang, L.-Y. Niu, Y. Zhang, Y.-Z. Chen, H.-B. Fu and Q.-Z. Yang, A solid-state fluorescent material based on carbazole-containing difluoroboron $\beta$-diketonate: multiple chromisms, the Self-Assembly behavior, and optical waveguides, Adv. Funct. Mater., 2017, 27, 1700332.

14 J. Luo, Z. Xie, J. W. Y. Lam, L. Cheng, B. Z. Tang, H. Chen, C. Qiu, H. S. Kwok, X. Zhan, Y. Liu and D. Zhu, Aggregationinduced emission of 1-methyl-1,2,3,4,5-pentaphenylsilole, Chem. Commun., 2001, 1740-1741.

15 G. Zhang, J. Lu and C. L. Fraser, Mechanochromic luminescence quenching: force-enhanced singlet-to-triplet intersystem crossing for iodide-substituted difluoroboron-dibenzoylmethane-dodecane in the solid state, Inorg. Chem., 2010, 49, 10747-10749.

16 Y. Lei, Y. Zhou, L. Qian, Y. Wang, M. Liu, X. Huang, G. Wu, H. Wu, J. Ding and Y. Cheng, Polymorphism and mechanochromism of $\mathrm{N}$-alkylated 1,4-dihydropyridine derivatives containing different electron-withdrawing end groups, J. Mater. Chem. C, 2017, 5, 5183-5192.

17 Z. Wang, Y. Li, D. Yuan, L. Qian, L. Li, H. Wu, M. Liu, J. Ding and X. Huang, The effect of molecular symmetry on the mechanofluorochromic properties of $4 \mathrm{H}$-pyran derivatives, Dyes Pigm., 2019, 162, 203-213.

18 Q. Qi, J. Qian, X. Tan, J. Zhang, L. Wang, B. Xu, B. Zou and W. Tian, Remarkable turn-On and color-tuned piezochromic luminescence: mechanically switching intramolecular charge transfer in molecular crystals, Adv. Funct. Mater., 2015, 25, 4005-4010.

19 J. M. Cole, M. A. Blood-Forsythe, T. C. Lin, P. Pattison, Y. Gong, A. Vazquez-Mayagoitia, P. G. Waddell, L. Zhang, N. Koumura and S. Mori, Discovery of S . C C identical with N intramolecular bonding in a thiophenylcyanoacrylate-based dye: realizing charge transfer pathways and dye $\cdots \mathrm{TiO}_{2}$ anchoring characteristics for dye-sensitized solar cells, ACS Appl. Mater. Interfaces, 2017, 9, 25952-25961.

20 Y. Xu, K. Wang, Y. Zhang, Z. Xie, B. Zou and Y. Ma, Fluorescence mutation and structural evolution of a $\pi$-conjugated molecular 
crystal during phase transition, J. Mater. Chem. C, 2016, 4, 1257-1262.

21 C. Wang and Z. Li, Molecular conformation and packing: their critical roles in the emission performance of mechanochromic fluorescence materials, Mater. Chem. Front., 2017, 1, 2174-2194.

22 L. Peng, Y.-N. Chen, Y. Qiang Dong, C. He and H. Wang, Surfactant-assisted self-assembled polymorphs of AIEgen di(4-propoxyphenyl)dibenzofulvene, J. Mater. Chem. C, 2017, 5, 557-565.

23 Y. Wang, G. Zhang, W. Zhang, X. Wang, Y. Wu, T. Liang, X. Hao, H. Fu, Y. Zhao and D. Zhang, Tuning the solid state emission of the carbazole and cyano-substituted tetraphenylethylene by co-crystallization with solvents, Small, 2016, 12, 6554-6561.

24 Q. Li and Z. Li, The Strong Light-Emission Materials in the aggregated state: what happens from a single molecule to the collective group, Adv. Sci., 2017, 4, 1600484.

25 S. Ohtani, M. Gon, K. Tanaka and Y. Chujo, A flexible, fused, Azomethine-Boron complex: thermochromic luminescence and thermosalient behavior in structural transitions between crystalline polymorphs, Chemistry, 2017, 23, 11827-11833.

26 R. Tan, Q. Lin, Y. Wen, S. Xiao, S. Wang, R. Zhang and T. Yi, Polymorphism and mechanochromic luminescence of a highly solid-emissive quinoline- $\beta$-ketone boron difluoride dye, CrystEngComm, 2015, 17, 6674-6680.

27 M. Louis, A. Brosseau, R. Guillot, F. Ito, C. Allain and R. Métivier, Polymorphism, mechanofluorochromism, and photophysical characterization of a carbonyl substituted difluoroboron$\beta$-diketone derivative, J. Phys. Chem. C, 2017, 121, 15897-15907.

28 H. Imoto, R. Fujii and K. Naka, 3,4-Diaminomaleimide Dyessimple luminophores with efficient orange-red emission in the solid state, Eur. J. Org. Chem., 2018, 837-843.

29 L. Wang, K. Wang, B. Zou, K. Ye, H. Zhang and Y. Wang, Luminescent chromism of boron diketonate crystals: distinct responses to different stresses, Adv. Mater., 2015, 27, 2918-2922.

30 P. Yadav, A. K. Singh, C. Upadhyay and V. P. Singh, Photoluminescence behaviour of a stimuli responsive Schiff base: Aggregation induced emission and piezochromism, Dyes Pigm., 2019, 160, 731-739. 